\title{
Attenuation of concentration fluctuations of water vapor and other trace gases in turbulent tube flow
}

\author{
W. J. Massman ${ }^{1}$ and A. Ibrom ${ }^{2}$ \\ ${ }^{1}$ US Forest Service, Rocky Mountain Research Station, 240 West Prospect, Fort Collins, CO 80526, USA \\ ${ }^{2}$ Bio Systems Department, Ris $\varnothing$ National Laboratory, DTU, Frederiksborgvej, 4000 Roskilde, Denmark
}

Received: 28 March 2008 - Published in Atmos. Chem. Phys. Discuss.: 28 May 2008

Revised: 8 September 2008 - Accepted: 8 September 2008 - Published: 28 October 2008

\begin{abstract}
Recent studies with closed-path eddy covariance (EC) systems have indicated that the attenuation of fluctuations of water vapor concentration is dependent upon ambient relative humidity, presumably due to sorption/desorption of water molecules at the interior surface of the tube. Previous studies of EC-related tube attenuation effects have either not considered this issue at all or have only examined it superficially. Nonetheless, the attenuation of water vapor fluctuations is clearly much greater than might be expected from a passive tracer in turbulent tube flow. This study reexamines the turbulent tube flow issue for both passive and sorbing tracers with the intent of developing a physicallybased semi-empirical model that describes the attenuation associated with water vapor fluctuations. Toward this end, we develop a new model of tube flow dynamics (radial profiles of the turbulent diffusivity and tube airstream velocity). We compare our new passive-tracer formulation with previous formulations in a systematic and unified way in order to assess how sensitive the passive-tracer results depend on fundamental modeling assumptions. We extend the passive tracer model to the vapor sorption/desorption case by formulating the model's wall boundary condition in terms of a physicallybased semi-empirical model of the sorption/desorption vapor fluxes. Finally we synthesize all modeling and observational results into a single analytical expression that captures the effects of the mean ambient humidity and tube flow (Reynolds number) on tube attenuation.
\end{abstract}

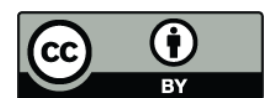

Correspondence to: W. J. Massman (wmassman@fs.fed.us)

\section{Introduction}

Eddy covariance technology (ECT) has been and continues to be critical to the quantification of exchange rates of $\mathrm{CO}_{2}$, $\mathrm{H}_{2} \mathrm{O}$, and other trace gases between the atmosphere and the terrestrial biosphere. The success and accomplishments of all global flux networks to date rests directly on ECT and the (sine qua non) technical capability to accurately measure the fluctuations in wind velocity and trace gases concentration. But no measurement technology is free of sources of instrument error and bias. It is well known, for example, that ECT underestimates the high frequency content of the fluctuations of these atmospheric variables as a result of finite response time of the instrumentation, spatial displacement of the sensors, line averaging effects for open-path instruments, and tube attenuation for closed-path instruments (e.g., Massman, 2000). Of particular interest to the present study are these frequency-dependent tube attenuation effects.

The first to address the attenuation of concentration fluctuations associated with sampling tubes was Philip (1963a,b), whose model-based study was focused exclusively on passive tracers and laminar tube flow. Later Lenschow and Raupach (1991), using water vapor as the tracer, measured the attenuation of concentration fluctuations associated with turbulent tube flows. In addition, they also developed a model of these frequency-dependent tube attenuation effects, the basis of which was the modeling and observational results of Taylor (1954). Surprisingly though when they compared the model predictions with the observed attenuation, they found that the attenuation of water vapor fluctuations is not only significantly greater than might be expected for a passive tracer, but also it is more strongly influenced by the flow Reynolds number than predicted as well. On the

Published by Copernicus Publications on behalf of the European Geosciences Union. 
other hand, Massman (1991), using a very different and presumably more complete model of turbulent tube flow, was successful at modeling the data of Lenschow and Raupach (1991). Consequently, the conundrum posed by the discrepancy of Lenschow and Raupach (1991) was assumed to have been resolved due to a better (or more physically realistic) model of turbulent tube flow. Nonetheless more recent observations by Clement (2004), Amman et al. (2006), and Ibrom et al. (2007) have suggested that the attenuation of atmospheric water vapor fluctuations is strongly influenced by relative humidity, which leads to the very likely possibility that some of the greater-than-expected attenuation observed by Lenschow and Raupach (1991) resulted in part from humidity effects. If so, this (a) invalidates the assumption, on which both Lenschow and Raupach (1991) and Massman (1991) are based, that water vapor is a passive tracer, and (b) clearly indicates a need to carefully reexamine the previous models of tube attenuation effects for passive tracers and to develop (if possible) a physically-based model that includes the effects of humidity on tube attenuation. Such is the intent and purpose of the present study.

Specifically this study takes a fresh look at the turbulent tube attenuation effects for passive scalars and develops a physically-based semi-empirical model that describes the additional attenuation associated with water vapor fluctuations. The physical processes associated with this additional attenuation are assumed to be related to sorption/desorption at the tube wall. Consequently, formulating the tube wall boundary condition for the trace gas tube transport equation requires developing a model of the sorptive wall fluxes. Massman (1991) showed that first-order sorption (or destruction) of ozone at the tube wall will result in additional attenuation in an eddy covariance ozone-flux sampling tube. Nevertheless, the present study attempts a very different formulation for the wall boundary condition in the hope that (at least some of) the results are generally applicable to any trace gas that might adhere to the inside surface of a tube (e.g., $\mathrm{H}_{2} \mathrm{O}, \mathrm{O}_{3}$, $\mathrm{NH}_{3}, \mathrm{SO}_{2}$, and many other polar molecules) and possibility to isotopes of such trace gases as well.

\section{Modeling scalar transport and the tube transfer func- tion}

The lateral and longitudinal dispersion of a tracer or solute being advective through a straight horizontal tube is described in terms of the advective-diffusive equation in cylindrical coordinates:

$$
\frac{\partial C}{\partial t}+U(r) \frac{\partial C}{\partial x}=\frac{1}{r} \frac{\partial}{\partial r}\left[r D(r) \frac{\partial C}{\partial r}\right]+D(r) \frac{\partial^{2} C}{\partial x^{2}}
$$

where $C=C(r, x, t)$ is the solute concentration, $r$ is the radial distance from the centerline of the tube, $x$ is the longitudinal distance from the mouth of the tube, $t$ is time, $U(r)$ is the radial profile of the longitudinal airstream velocity, and $D(r)$ is the radial profile of the turbulent diffusivity. (NOTE: For reasons that will become clearer later it is more convenient to express all radial dependencies in terms of the dimensionless wall coordinate, $\rho$, rather than in terms of $r$; here $\rho=1-r / a$ and $a$ is the radius of the tube.) To obtain the tube transfer function, which characterizes the tube's attenuation effects, requires a solution to Eq. (1). The present study employs the spectral decomposition/eigenvalue approach by assuming that

$C(\rho, x, t)=\widehat{C}_{\lambda}(\rho) e^{i \omega(t-\lambda x / \bar{U})}$

where $i=\sqrt{-1}$ is the unit imaginary number, $\omega$ is circular frequency (radians s ${ }^{-1}$ ), $\bar{U}$ is the cross-sectionally averaged $U(\rho), \lambda$ is the eigenvalue (a complex number with both real and imaginary parts), and $\widehat{C}_{\lambda}(\rho)$ is the eigenfunction, which is also complex-valued. In the most general terms the solution to Eq. (1) is now synonymous with finding the eigenvalue, which directly determines the tube transfer function.

Substituting Eq. (2) into Eq. (1) and after some algebraic manipulations Eq. (1) can be transformed into the following equation:

$$
\begin{aligned}
\frac{1}{1-\rho} \frac{d}{d \rho}[ & \left.(1-\rho) G_{D}(\rho) \frac{d \widehat{C}_{\lambda}(\rho)}{d \rho}\right]= \\
& \Omega\left[i-i \lambda G_{U}(\rho)+\gamma \lambda^{2} \Omega G_{D}(\rho)\right] \widehat{C}_{\lambda}(\rho)
\end{aligned}
$$

where $\Omega=a^{2} \omega / D(1)$ and $D(1)$ is the centerline value of the turbulent diffusivity (discussed more later), $G_{U}(\rho)=U(\rho) / \bar{U}, G_{D}(\rho)=D(\rho) / D(1)$, and $\gamma$ is a flow related parameter; $\gamma=4 D^{2}(1) v^{-2} R e^{-2}$, where $R e=2 a \bar{U} / v$ is the tube flow Reynolds number and $v$ is the molecular viscosity of air. (Note for the present purposes $v$ can be considered a constant. But, in fact, it is a function of both temperature and relative humidity (e.g., Studnikov, 1970; Tsilingiris, 2008). In general terms these two effects will tend to add "noise" or uncertainty to specific model predictions. Nonetheless, for typical environmental conditions encountered at eddy covariance sites and within closed-path eddy covariance tubes the relative humidity effects can probably be disregarded, but the (larger) temperature effects may influence the Reynolds number and the turbulent diffusivity (see next section) enough during a daily or seasonal cycle that they might need to be considered when employing or evaluating specific model predictions.)

Except for a slight change in notation, this last equation is identical to Eq. (3) of Massman (1991). But at this point the present development diverges significantly from Massman (1991). Here we take very different approaches to modeling the turbulent diffusivity, $D(\rho)$, and to solving for the eigenvalue and we employ a (somewhat) different model for $U(\rho)$. By choosing approaches that contrast strongly with Massman (1991), we hope to better understand how different methodologies can quantitatively impact model predictions. 


\subsection{Modeling $U(\rho)$}

To model $U(\rho)$ we adapt the model of $U(\rho)$ given by Eq. (1.6.10) on page 35 of Polyanin et al. (2002). Specifically, we assume

$$
\frac{U(\rho)}{\bar{U}}=\frac{G(\rho)}{2 \int_{0}^{1}(1-\rho) G(\rho) d \rho}
$$

where

$$
\begin{aligned}
& G(\rho)=\ln (1+0.4 K \rho)+\ln \frac{1.5(2-\rho)}{1+2(1-\rho)^{2}}+ \\
& 3\left[1-e^{-0.1 K \rho}-0.1 K \rho e^{-0.3 K \rho}\right]
\end{aligned}
$$

with $K$ as the Karman number, $K=a u_{*} / \nu$, and $u_{*}$ is the wall friction velocity. Here we should note that the cross sectional average of any variable, $Q(\rho)$, is given as $\bar{Q}=2 \int_{0}^{1}(1-\rho) Q(\rho) d \rho$ and that by definition $u_{*} / \bar{U} \equiv 2 K / R e$. To complete the model for $U(\rho)$ we relate $K$ and $u_{*} / \bar{U}$ to $R e$ by employing the Blasius relation (e.g., McKeon et al., 2005), which is $8\left(u_{*} / \bar{U}\right)^{2}=0.3164 R e^{-1 / 4}$. This yields $K=\sqrt{0.3164 / 32} R e^{7 / 8} \quad$ and $\quad u_{*} / \bar{U}=0.1989 R e^{-1 / 8}$, which are important model constitutive relationships, especially for modeling $D(\rho)$.

The present model of $U(\rho)$ was chosen over the one originally adopted by Massman (1991) for a very important reason. On one hand, the new model is just as physically realistic (if not more so) than Massman's original model of $U(\rho)$, but, on the other, it has fewer (in fact no) empirical parameters. Consequently, the new model is specifically intended to make it more difficult to obscure the need for modeling the adsorption/desorption processes by simply adjusting velocity profile parameters to fit observations. This philosophy of minimizing the number (and sensitivity) of model parameters carries over into the next section, which outlines models of turbulent diffusivity that are not dependent on the velocity shear $(\partial U / \partial \rho)$ or mixing length theory.

\subsection{Modeling $D(\rho)$}

Massman (1991)'s model for $D(\rho)$ has a significant conceptual problem, i.e., as the turbulent diffusivity becomes small as the centerline is approached $\left(\lim _{\rho=1} D(\rho) \approx 0\right)$. This is a consequence of using mixing length theory, which parameterizes the turbulent diffusivity in terms of the velocity shear $(\partial U / \partial \rho \rightarrow 0$ as $\rho \rightarrow 1)$. This concern is not new (e.g., Reichardt, 1951). Consequently, the present study develops two rather different parameterizations of $D(\rho)$, both of which circumvent this conceptual problem. These two models are used to explore the model's sensitivity to different formulations of $D(\rho)$.

From Taylor (1954) we know that $\bar{D}=10.1 a u_{*}$, which can be written as $\bar{D}=10.1 K \nu=R e^{7 / 8} v$. Therefore, we will construct a function, $F(\rho)$, such that $D(\rho)=\bar{D} F(\rho)$ and $\bar{F}=1$.
Since it is not unreasonable to assume that the turbulent diffusivity is proportional to the turbulent viscosity, $v_{T}$, we construct $F(\rho)$ from models of $\nu_{T}(\rho)$.

The first model assumes that $F(\rho) \propto U(\rho) / \bar{U}$ (e.g., Kirkegaard and Kristensen, 1996). Such an assumption is plausible because it not only eliminates the logical contradictions associated with using mixing length theory near the tube centerline, but it may also be theoretically justifiable for bounded flows, for which $v_{T}(\rho) \sim U(\rho)$ has been suggested, (e.g., Hussein et al., 1994; Pope, 2000). Nevertheless, despite its appealing simplicity this modeling assumption is not complete, because near the tube wall (i.e., as $\rho \sim 0$ ) this assumption combined with $G(\rho)$ from Eq. (4) suggests that the Reynolds stresses $\sim v_{T}(\rho) \partial U / \partial \rho \sim U(\rho) \partial U / \partial \rho \sim \rho$, which differs than the expected result of $\sim \rho^{3}$ (e.g., Kim et al., 1987; Pope, 2000). To compensate $F(\rho) \propto G(\rho) V(\rho)$ is assumed, where

$V(\rho)=1-e^{-A K \rho^{2}}$

is a modified version of the original van Driest function (van Driest, 1956), $1-e^{-K \rho / A^{+}}$, in which the exponent is linearly dependent on $\rho$. Our modification to the original van Driest function ensures the functional description of $\sim \rho^{3}$ for the Reynolds stresses near the tube wall. For the present study $A=0.0375$ in accordance with the original van Driest parameter $A^{+}=26-28$ (e.g., Pope, 2000; Rusak and Meyerholz, 2006). Although the van Driest function is somewhat empirical, it is not without logic or precedent (Rusak and Meyerholz, 2006). Furthermore, by a careful choice of the parameter, $A$, the turbulent viscosity can be made to display a broad uniform maximal value near the tube centerline, which captures the functional dependence suggested from several observation-based studies (Pope, 2000).

The first model for $D(\rho)$ is given as:

$D(\rho) / \nu=\left(R e^{7 / 8}-S c^{-1}\right) \frac{G(\rho) V(\rho)}{\overline{G V}}+S c^{-1}$

where $S c$ is the Schmidt number and the single $S c^{-1}$ (rightmost) term is included to account for molecular diffusion, which will dominate turbulent diffusion very near the wall (i.e., $D(\rho) / \nu \sim S c^{-1}$ when $\rho \sim 0$ ). The multiplier $\left(R e^{7 / 8}-S c^{-1}\right)$ on the left side is used to maintain the equality $\bar{D} / \nu=R e^{7 / 8}$ in accordance with Taylor (1954). (We should note, primarily for the sake of completeness, that the constructed function, $F(\rho)=R e^{-7 / 8} D(\rho) / \nu$.)

The second model of $D(\rho)$ is adapted from Reichardt (1951)'s model of turbulent viscosity, which assumes that $v_{T}(\rho) / v \propto \rho(2-\rho)\left[1+2(1-\rho)^{2}\right]=\left(2 \rho-\rho^{2}\right)\left(3-4 \rho+2 \rho^{2}\right)$.

This empirical function displays a local maximum at $\rho=0.5$ and a shallow minimum near the tube centerline. This centerline minimum is only somewhat less than the local maximum so that $D(1) \gg 0$ (e.g., Kays and Crawford, 1993, p. 247). This model of $v_{T}(\rho)$ is reasonable because there is no production of turbulence at the centerline (where 
there is no velocity shear). Consequently, turbulence is continuously diffusing toward the centerline from the nearby high shear regions (where it is being generated) and it is being continuously dissipated near the centerline at the same rate.

This study enhances Reichardt (1951)'s original model with a parameter (here termed Reichardt's parameter) that allows the position of the local maximum to vary somewhat from $\rho=0.5$, in accordance with observationally-based inferences (e.g., Sherwood et al., 1975). This parameter is denoted by $B$ in the following expression:

$E(\rho)=\left(2 \rho-\rho^{2}\right)\left(B-4 \rho+2 \rho^{2}\right)$

where $2.25 \leq B \leq 4.0$ (with $E_{\max }$ occuring at $\left.\rho_{\max }=1-0.5 \sqrt{4-B}\right)$ and $E(\rho)$ is used to define the second model of $D(\rho)$ as follows:

$D(\rho) / \nu=\left(R e^{7 / 8}-S c^{-1}\right) \frac{E(\rho) V(\rho)}{\overline{E V}}+S c^{-1}$

The van Driest function, $V(\rho)$, is included in this model of $D(\rho)$ for the same reason it is included the first model, Eq. (5). But including $V(\rho)$ does alter the position of the maximum value of $D(\rho)$ relative to $E_{\max }$. Nonetheless, $\rho_{\max }=1-0.5 \sqrt{4-B}$ remains a very good approximation for estimating the position of $D_{\max } / v$ providing $B \geq 2.25$.

\subsection{Method of solution}

Solving Eq. (3) by numerical methods is possible, but many of the numerical complexities and precision issues can be significantly reduced by simplifying Eq. (3) using a perturbation-expansion technique.

The first step in this procedure requires establishing that for eddy covariance applications $\Omega<1$ is valid. Recalling that $\Omega=a^{2} \omega / D(1)$ it follows that $\Omega=\left(a^{2} \omega / v\right)\left(R e^{-7 / 8} \Delta_{D}^{-1}\right)$, where $\Delta_{D}$ is defined by the relation $D(1) / \nu=R e^{7 / 8} \Delta_{D}$. In general $\Delta_{D}$ is a function of $A, R e$, and $S c^{-1}$, but for the present study it is sufficient to note that $\Delta_{D}$ is a monotonically decreasing function of $R e$ such that $1.38 \leq \Delta_{D} \leq 3.34$. Next, assuming $v \approx 0.15 \mathrm{~cm}^{2} \mathrm{~s}^{-1}$, that the tube diameter is not much larger than about $1 \mathrm{~cm}$ (i.e., $a^{2} \leq 0.25 \mathrm{~cm}^{2}$ ), that the highest frequency of interest for eddy covariance is likely to be a sampling frequency of about $20 \mathrm{~Hz}$ (i.e., $\omega \leq 2 \pi \times 20 \mathrm{~s}^{-1}$ ), and that the minimum value for $R e$ is about 2300 (i.e., $R e^{-7 / 8} \leq 1.144 \times 10^{-3}$ ), it follows that $\Omega<0.14<1$. These results are relevant because we can now quite accurately approximate $\widehat{C}_{\lambda}(\rho)$ and $\lambda$ as follows:

$\widehat{C}_{\lambda}(\rho)=\widehat{C}_{0}(\rho)+\Omega \widehat{C}_{1}(\rho)+\Omega^{2} \widehat{C}_{2}(\rho)+\Omega^{3} \widehat{C}_{3}(\rho)$

and

$\lambda=1+\alpha_{1} \Omega+\alpha_{2} \Omega^{2}$
Substituting these expressions into Eq. (3) and equating powers of $\Omega$ yields a set of recursive ordinary differential equations (ODEs) for $\widehat{C}_{0}(\rho), \widehat{C}_{1}(\rho), \widehat{C}_{2}(\rho)$, and $\widehat{C}_{3}(\rho)$, in which $\Omega$ does not directly appear.

The next step is to formulate the boundary conditions and solve the ODEs sequentially for $\widehat{C}_{0}(\rho), \widehat{C}_{1}(\rho), \alpha_{1}, \widehat{C}_{2}(\rho)$, and $\alpha_{2}$ as functions of the boundary conditions and the characteristics of the profiles $U(\rho)$ and $D(\rho)$. Most significant to the present discussion is that $\alpha_{1}$ is an imaginary number of the form $\alpha_{1}=-i\left|\alpha_{1}\right|$, where $\left|\alpha_{1}\right|$ is the modulus of $\alpha_{1}$, and $\alpha_{2}<0$ is real. Here $\alpha_{1}$ is the imaginary part of the eigenvalue, $\lambda$, and $\alpha_{2}$ is its real part. Relative to the transfer function (discussed in the next section) $\alpha_{1}$ is that part of the eigenvalue that relates to the attenuation of the trace gas fluctuations, whereas $\alpha_{2}$ relates to the frequency-dependent phase shift or lag time associated with the tube flow.

The boundary condition at the center of the tube requires that there be no net exchange of mass (no flux) across the centerline of the tube. Consequently, for $n=0,1,2, \ldots,\left\{G_{D}(\rho) d \widehat{C}_{n} / d \rho\right\}_{\rho=1}=0$, which implies that $\left\{d \widehat{C}_{n} / d \rho\right\}_{\rho=1}=0$ since $G_{D}(1) \neq 0$. For a passive tracer the appropriate boundary condition at the tube wall is again no net flux, i.e., $\left\{G_{D}(\rho) d \widehat{C}_{n} / d \rho\right\}_{\rho=0}=0$, which implies that $\left\{d \widehat{C}_{n} / d \rho\right\}_{\rho=0}=0$ since $G_{D}(0) \neq 0$. With these boundary conditions the solution $\widehat{C}_{0}(\rho)$ is $\widehat{C}_{0}(\rho)=\mathcal{C}$, a constant. With no loss of generality $\widehat{C}_{0}(\rho) \equiv 1$ can be assumed for a passive tracer. Unfortunately, solutions for $\widehat{C}_{n}(\rho) \quad(n \geq 1)$ cannot be found analytically and so must be computed numerically. This is done using a modified shooting method with a fourth-order Runge-Kutta algorithm with an adaptive step size (Press et al., 1992) to solve the ODEs for $\widehat{C}_{1}(\rho)$ and $\widehat{C}_{2}(\rho)$ numerically and an algebraic/numerical procedure for determining $\alpha_{1}$ and $\alpha_{2}$. The integration procedure assumes that $\widehat{C}_{n}(0)=0 \quad(n \geq 1)$. Nevertheless, the numerical procedures cannot directly handle the singularity at the centerline $(\rho=1)$ that is typical of these ODEs and Eq. (3). This singularity is treated by matching the numerical solution (at some point near but not at the centerline) with a power series expansion of the form $\lim _{\rho \rightarrow 1} \widehat{C}_{n}(\rho)=a_{0 n}+a_{1 n}(1-\rho)+a_{2 n}(1-\rho)^{2}+a_{3 n}(1-\rho)^{3}+$ $\cdots$, where the coefficients can be determined analytically from the appropriate ODE. Finally, it is not necessary to solve for $\widehat{C}_{3}(\rho)$ explicitly, because the only relevant information required to determine $\alpha_{2}$ is the boundary condition at the tube wall.

\subsection{The tube transfer function}

Once $\alpha_{1}$ and $\alpha_{2}$ been determined the transfer function, $h(\omega)$, for a tube of length $L$ can be determined from Eq. (2) as:

$h(\omega)=\frac{\widehat{C}(\rho, L, t)}{\widehat{C}_{\lambda}(\rho) e^{i \omega t}}=e^{-i \omega \lambda L / \bar{U}}$ 
which can now be expressed as

$h(\omega)=\left[e^{-\Lambda_{1} \omega^{2} a L / \bar{U}^{2}}\right]\left[e^{-i(\omega L / \bar{U})\left(1-\Lambda_{2} \omega^{2} a^{2} / \bar{U}^{2}\right)}\right]$

where, borrowing the notation from Massman (1991), $\Lambda_{1}=0.5\left|\alpha_{1}\right| \Delta_{D}^{-1} R e^{1 / 8}$ and $\Lambda_{2}=0.25\left|\alpha_{2}\right| \Delta_{D}^{-2} R e^{1 / 4}$.

The first term in brackets on the RHS of Eq. (8) is the real-valued transfer function, $H(\omega)$, associated with the attenuation of fluctuations and the second term in brackets on the RHS expresses the phase shift (or tube lag time) (e.g., Massman, 2000, 2004). Therefore,

$H(\omega)=e^{-\Lambda_{1} \omega^{2} a L / \bar{U}^{2}}$

and

$h_{\text {phase }}(\omega)=e^{-i(\omega L / \bar{U})\left(1-\Lambda_{2} \omega^{2} a^{2} / \bar{U}^{2}\right)}$

where the tube lag time is $L / \bar{U}\left(1-\Lambda_{2} \omega^{2} a^{2} / \bar{U}^{2}\right)$. Note that the tube lag time is usually assumed to be $L / \bar{U}$, which is correct only for those frequencies such that $\Lambda_{2} \omega^{2} a^{2} / \bar{U}^{2} \ll 1$. Assessing the importance of the second order term, $\Lambda_{2} \omega^{2} a^{2} / \bar{U}^{2}$, requires evaluating the validity of this inequality. This is accomplished by noting that $\omega^{2} a^{2} / \bar{U}^{2}=4 \omega^{2} a^{4} R e^{-2} / v^{2}$ and then using the same inequalities and values for $\omega, a, R e$, and $v$ that were used to establish that $\Omega<1$. The resulting calculations show that $\Lambda_{2} \omega^{2} a^{2} / \bar{U}^{2}<\Lambda_{2} / 30$. Therefore, one should expect that $\Lambda_{2} / 30<1$ and that this condition should be considered when assessing any particular model's performance.

Before presenting the solutions, $\Lambda_{1}=\Lambda_{1}(R e)$ and $\Lambda_{2}=\Lambda_{2}(R e)$, it is worthwhile to re-examine the modeling results of Lenschow and Raupach (1991) and Kirkegaard and Kristensen (1996), which is done in the next section. The benefit and the intent behind these next two approaches is to avoid (or to reduce as much as possible) the need to specifically model $D(\rho)$, which is at best highly uncertain and at worst completely unknown.

\section{Other modeling strategies}

\subsection{Taylor (1954)'s approach}

The model for the attenuation coefficient $\Lambda_{1}$ developed by Lenschow and Raupach (1991) is basically a restatement of Taylor (1954). This section, which follows Kristensen and Kirkegaard (personal communication, 2007), calculates $\Lambda_{1}$ and $\Lambda_{2}$ from Taylor (1954)'s model. This begins with a simplified version of Eq. (1), namely:

$\frac{\partial \bar{C}}{\partial t}+\bar{U} \frac{\partial \bar{C}}{\partial x}=\bar{D} \frac{\partial^{2} \bar{C}}{\partial x^{2}}$

where $\bar{C}=\bar{C}(x, t)$ is the cross-sectionally averaged concentration. Next $\bar{C}(x, t)=\widehat{C}(x) \exp (i \omega t)$ is assumed. Using the dimensionless variable $\eta=x / a$ and Taylor (1954)'s relationship for $\bar{D}$ (i.e., $\bar{D} / \nu=R e^{7 / 8}$ ), this simplified tube flow equation is expressed in dimensionless form as

$\frac{d^{2} \widehat{C}}{d \eta^{2}}-\sigma \frac{d \widehat{C}}{d \eta}-i \Omega_{T} \widehat{C}=0$

where $\sigma=0.5 R e^{1 / 8}$ and $\Omega_{T}=\omega a^{2} R e^{-7 / 8} / \nu=0.5 R e^{1 / 8}[\omega a / \bar{U}]$.

The exponentially decaying solution to this last equation is the transfer function

$\widehat{C}(\eta)=\widehat{C}_{0} e^{s \eta}$

where $s=\left(\sigma-\sqrt{\sigma^{2}+4 i \Omega_{T}}\right) / 2$. This expression for $s$ can be further simplified by noting that $4 \Omega_{T} \ll \sigma^{2}$ for most eddy covariance applications. This is basically equivalent to perturbation assumption $\Omega<1$ previously discussed and employed with the eigenvalue model above. This $\Omega_{T}$ inequality allows $s$ to be expanded in a Taylor's series, which when truncated after 4 terms yields the following final solution for $\bar{C}(L, t)$ :

$$
\begin{aligned}
\bar{C}(L, t)=\widehat{C}_{0} e^{i \omega t} & {\left[e^{-\left\{2 R e^{-1 / 8}\right\}\left(\omega^{2} a L / \bar{U}^{2}\right)}\right] \times } \\
& {\left[e^{-i(\omega L / \bar{U})\left(1-\left\{8 R e^{-1 / 4}\right\} \omega^{2} a^{2} / \bar{U}^{2}\right)}\right] }
\end{aligned}
$$

from which the attenuation coefficients $\Lambda_{1 T}=2 R e^{-1 / 8}$ and $\Lambda_{2 T}=8 R e^{-1 / 4}$ are easily identified.

\subsection{Separation of variables}

The tube flow model developed by Kirkegaard and Kristensen (1996) is based on the assumption that $D(\rho) \propto U(\rho)$, which allows Eq. (1) to be solved by separation of variables. This section examines the separation of variables technique for solving the tube transport equation to calculate the resulting attenuation coefficients $\Lambda_{1 K}=\Lambda_{1 K}(R e)$ and $\Lambda_{2 K}=\Lambda_{2 K}(R e)$. But the present development is somewhat different from that employed by Kirkegaard and Kristensen (1996) and the full mathematical development, which is fairly involved, will only be outlined and summarized here. (Note the symbols used in this section are consistent with their usage throughout this study.) The first assumption is

$C(\rho, x, t)=\widehat{C}(\rho, x) e^{i \omega t}$

The second assumption is that the a ratio $U(\rho) /\left[\bar{U} G_{D}(\rho)\right]$ is well approximated by its cross sectional average, i.e., $U(\rho) /\left[\bar{U} G_{D}(\rho)\right]=\overline{U(\rho) /\left[\bar{U} G_{D}(\rho)\right]} \equiv \beta$. Without this, or a similar assumption, separation of variables is not possible. (Note this assumption yields a slightly different value for $\overline{U(\rho) /\left[\bar{U} G_{D}(\rho)\right]}$ than the equivalent parameter used by Kirkegaard and Kristensen, 1996.) 


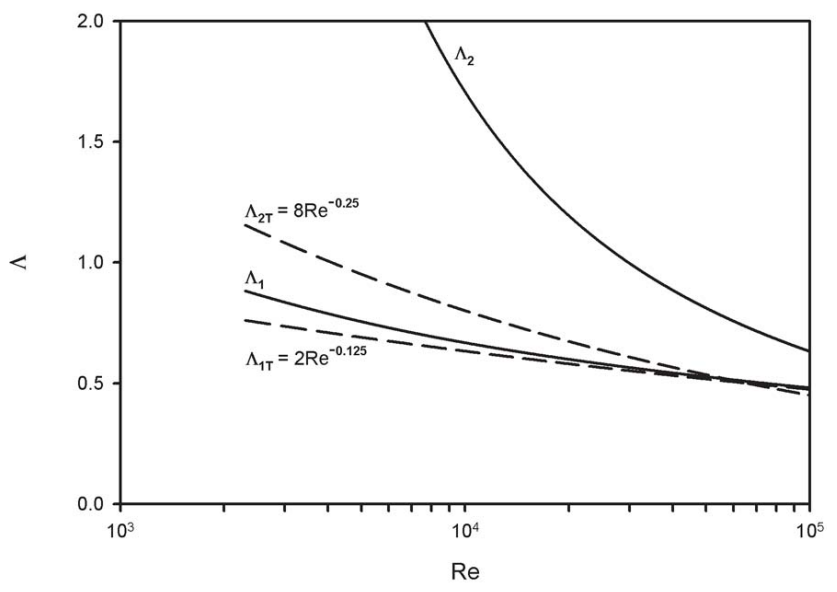

Fig. 1. Transfer function attenuation coefficients $\Lambda_{1}, \Lambda_{2}, \Lambda_{1 T}$, and $\Lambda_{2 T} \cdot \Lambda_{1}$ and $\Lambda_{2}$ are first and second order solutions to the eigenvalue model Eqs. (3), (5), and (8). $\Lambda_{1 T}$ and $\Lambda_{2 T}$ are Taylor's solution as shown in Eq. (14). Not included here is $\Lambda_{1 K}$, the solution to the separation-of-variables model of Kirkegaard and Kristensen (1996), which yielded $\Lambda_{1 K} \leq 0.3 \Lambda_{1 T}$.

Equation (1) can now be written as

$$
\begin{aligned}
\frac{1}{G_{D}(\rho)(1-\rho)} \frac{\partial}{\partial \rho} & {\left[(1-\rho) G_{D}(\rho) \frac{\partial \widehat{C}}{\partial \rho}\right]-\frac{i \Omega}{G_{D}(\rho)} \widehat{C}=} \\
& -\frac{\partial^{2} \widehat{C}}{\partial \eta^{2}}+\sigma \frac{\partial \widehat{C}}{\partial \eta}
\end{aligned}
$$

where $\sigma=0.5 R e^{1 / 8} \Delta_{D}^{-1} \beta$ and $\beta$ is a monotonically decreasing function of $R e$ such that $34.3 \leq \beta \leq 21.7$ (for the first model of $D(\rho)$, which is sufficient for the present purposes). Assuming the variables $\eta$ and $\rho$ are separable, i.e., that $\widehat{C}(\eta, \rho)=\widehat{A}_{\lambda}(\rho) \widehat{B}_{\lambda}(\eta)$, then Eq. (16) can be partitioned into two ODEs, one for $\widehat{A}_{\lambda}(\rho)$ and one for $\widehat{B}_{\lambda}(\eta)$; where $-i \lambda$ is the constant of separation and $\lambda$ is an eigenvalue. But $\lambda$ it is not necessarily numerically the same as the eigenvalue above; nor is it possible to assume that the eigenfunction $\widehat{A}_{\lambda}(\rho)$ is the same as $\widehat{C}_{\lambda}(\rho)$ above. At this point solving for the eigenvalue proceeds much as discussed above for the solution to Eq. (3) and the transfer function is determined from the solution for $\widehat{B}_{\lambda}(\eta)$.

For the present purposes it is sufficient to summarize the separation-of-variables model from the $\Lambda_{1}$ results alone. Either version of $D(\rho)$ yields the following inequality for $\Lambda_{1 K}$ :

$\Lambda_{1 K} \leq 0.3 \Lambda_{1 T}$

which clearly suggests that the separation of variables approach (as outlined here) predicts much less attenuation than Taylor (1954)'s model.

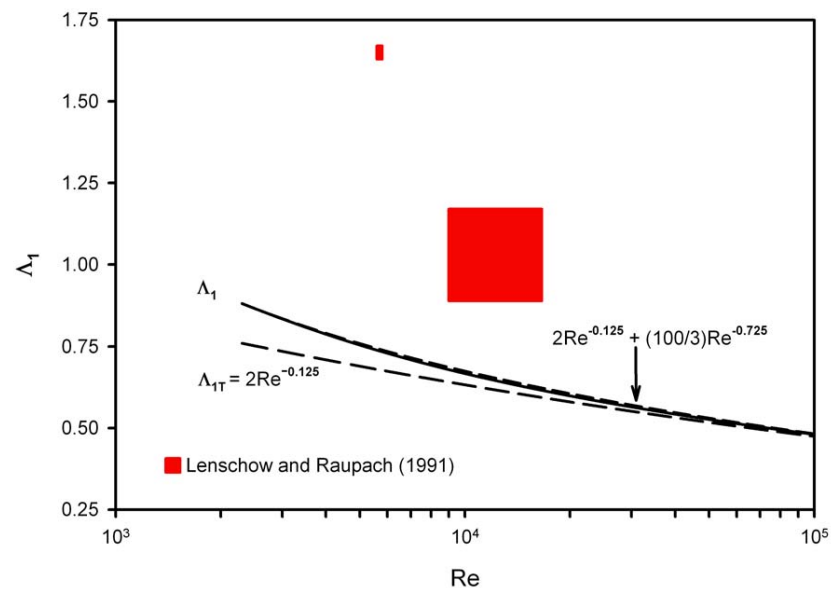

Fig. 2. Comparison of modeled (passive tracer) attenuation coefficients with a summary of some experimental observations associated with water vapor fluctuations from Lenschow and Raupach (1991) as determined by Massman (1991).

\section{Attenuation of a passive tracer}

Figure 1 shows the first and second order attenuation coefficients as functions of the tube flow Reynolds number for the eigenvalue model and Taylor (1954)'s model. Comparing $\Lambda_{1}(R e)$ with $\Lambda_{1 T}(R e)$ and $\Lambda_{2}(R e)$ with $\Lambda_{2 T}(R e)$ suggests that Taylor (1954)'s approximation is reasonable for the first order term, $\Lambda_{1}$, but that it may not be so for the second order term, $\Lambda_{2}$. Nevertheless, assuming that the eigenvalue model produces a more precise estimate for the attenuation coefficients of a passive tracer, $\Lambda_{1}(R e)$ can be approximated to within $\pm 1 \%$ by the analytical expression $2 R e^{-1 / 8}+(100 / 3) R e^{-0.725}$ (shown in Fig. 2). The attenuation term $(100 / 3) R e^{-0.725}$ largely results from the van Driest function, $V(\rho)$, and we interpret it to be the attenuation associated with the momentum boundary layer of the tube wall. By using a cross-sectionally averaged formulation, Taylor (1954)'s model would have eliminated this additional attenuation. It should be noted here that the maximum value for $\Lambda_{2}$ produced by the present eigenvalue model, which extends beyond the range of the $y$-axis of Fig. 1 , is about 4.7 so that $\Lambda_{2} / 30<0.16$, thereby confirming the earlier analysis for the first model of the turbulent diffusivity, Eq. (5).

The eigenvalue model with the second parameterization for the turbulent diffusivity, Eq. (6), produced estimates of $\Lambda_{1}(R e)$ that were within about $\pm 4 \%$ of the (aforementioned) analytical expression. But, the $\Lambda_{2}(R e)$ associated with this second formulation of the turbulent diffusivity displayed a significant sensitivity to the position of $D_{\max } / v$. So much so that as $B$ varied across its range of values the associated $\Lambda_{2}(R e)$ varied by more that a factor of two relative to $\Lambda_{2}(R e)$ shown in Fig. 1. Clearly these results suggest that $\Lambda_{2}(R e)$ is quite sensitive to the shape of $D(\rho)$, which leads to the idea of designing an observational experiment to 
exploit this sensitivity, thereby obtaining a better empirical understanding and model parameterization of $D(\rho)$.

Although the agreement between the present eigenvalue model and Taylor (1954)'s model is reasonably satisfying, the present results are not in agreement with the observations of Lenschow and Raupach (1991) or the modeling results of Massman (1991). Figure 2 shows $\Lambda_{1}(R e)$, its analytical approximation, and $\Lambda_{1 T}(R e)$ with a summary of the data (red boxes) from Lenschow and Raupach (1991) for the regions $5600 \leq R e \leq 5900$ and $9000 \leq R e \leq 16600$. These observational data clearly show much more attenuation than predicted by any of the present models. On the other hand, Massman (1991)'s model (not shown in Fig. 2) quite successfully predicted the observed attenuation. This disparity leads to two conclusions:

(I) The additional attenuation observed by Lenschow and Raupach (1991) results from the sorption/desorption of water molecules onto the (brass) tube walls. Therefore, none of the present passive-tracer models, which assume no interaction at the tube walls, are able to account for this additional attenuation. Consequently, the assumption, made by both Lenschow and Raupach (1991) and Massman (1991), that water vapor can be used as passive tracer (even under presumed ideal or 'equilibrium' conditions), is likely false. This last conclusion should not be too surprising given the recent observations of how strongly ambient relative humidity affects the attenuation of water vapor fluctuations in closedpath eddy covariance systems (e.g., Clement, 2004; Amman et al., 2006; Ibrom et al., 2007).

(II) Massman (1991)'s original model is sufficiently robust (possibly by happenstance) that it is able to capture the variation displayed by the data of Lenschow and Raupach (1991). Consequently, this earlier model was successful at least in part because of the formulations for $U(\rho)$ and $D(\rho)$, which are very different than those used in the present study. These earlier formulations included two parameters, which Massman tuned to fit the data of Lenschow and Raupach (1991). On the other hand, $\Lambda_{1}(R e)$ from the present turbulent tube flow model (which also contains two "adjustable" parameters, the van Driest parameter $A$ and Reichardt's parameter $B$ ) is not as sensitive to variations of these parameters. For example, the present attenuation coefficient $\Lambda_{1}(R e)$ is at most only weakly sensitive to (even large variations in) $A$ or $B$ and then only in the region $2300 \leq R e \leq 4000$. This is not entirely accidental because we sought to improve on Massman (1991)'s original model of $D(\rho)$ by developing models that were not only more realistic, but that also had fewer adjustable parameters. An important aspect of this is that the resulting model is less sensitive to variations of those parameters and the attenuation coefficients are less dependent on the details of the model assumptions.

\section{Attenuation of a sorbing/desorbing tracer}

For a trace gas interacting with the tube wall, the wall boundary condition requires a mathematical formulation of the physical processes that describe the mass fluxes associated with near-wall turbulent transport and the sorption/desorption onto the tube wall. It should not be surprising that a general formulation of this boundary condition could be quite complex because the physical processes at the tube wall are physiochemical in nature and molecular in scale and involve various aspects of the kinetic theory of gases, thin film dynamics, phase changes (condensation and evaporation) on clean homogeneous surfaces and on internal tube surfaces contaminated with atmospheric aerosols, as well as the dynamics of near-wall turbulent boundary layer effects. For application to the present study many of these processes are unknowable (at the very least) and so cannot be quantified with much certainty. Nevertheless, the approach taken here begins with a description of a comprehensive model of the mass fluxes to the tube wall, which is then simplified to produce a physically-based semi-empirical model of the wall boundary condition. We take this approach in order to gain insight into the physical processes involved and some ability into their quantification for modeling application. Once completed the wall boundary condition is then used with the turbulent tube flow model (described above) to test how well the model reproduces the results of Lenschow and Raupach (1991). The final section discusses the (unexpected) empirical adjustments that the model requires and then presents a single analytical expression for the attenuation coefficient ( $\Lambda_{1}$ only) that best synthesizes the present model and the observational results of Lenschow and Raupach (1991) and Ibrom et al. (2007).

\subsection{Wall boundary condition}

\subsubsection{Near-wall turbulent mass flux}

The turbulent mass flux, $F_{C}$, is often parameterized as: $F_{C}=h_{C} u_{*}\left(C_{\infty}-C_{\text {surf }}\right)$, where $h_{C}$ is the mass transfer coefficient through the tube wall's turbulent boundary layer (for water vapor Brutsaert, 1982, equates $h_{C}$ with the Dalton number), $C_{\infty}$ is the trace gas concentration within the mean flow region of the tube somewhere well away from the influence of the quasi-laminar wall boundary layer, and $C_{\text {surf }}$ is the gas concentration at the surface of the tube wall, which in wall coordinates is equivalent to $C(0)$. Since $F_{C}$ is the same as the diffusional wall flux $\left(F_{C}=-D_{g} d C / d r\right)$, the gradient of $C(r)$ at the wall in the wall coordinate $\rho$ is $C^{\prime}(0)=\{d C / d \rho\}_{\rho=0}=h_{C} K S c\left(C_{\infty}-C(0)\right)$. Several empirical models have been developed for (a smooth-wall) $h_{C}$ for both heat and mass transfer (e.g., Aravinth, 2000), many of which yield similar results - at least for the present application and set of trace gases, which can be characterized by $S c \approx 1$ or $P r \approx 1$ where $P r$ is the Prandtl number. Adapting 
the model of Pinczewski and Sideman (1974) for $h_{C}$, which is $h_{C}=0.064\left(u_{*} / \bar{U}\right) S c^{-1 / 2}\left(1.1+0.44 S c^{-1 / 2}-0.70 S c^{-1 / 6}\right)$, yields the following approximate relationship: $h_{C} K S c \approx 0.00108 R e^{3 / 4} S c^{1 / 2}$, which will be denoted by $\kappa_{b}$. Therefore, for the present study

$C^{\prime}(0)=\kappa_{b}\left\{C_{\infty}-C(0)\right\}$

and $\kappa_{b}=\left(1.08 \times 10^{-3}\right) R e^{3 / 4} S c^{1 / 2}$ is the dimensionless form of the turbulent boundary layer conductance associated with the tube wall.

Superficially Eq. (18) may appear adequate for the present purposes; but unfortunately, $C(0)$ cannot be specified solely on the basis of turbulent tube flow dynamics. In general, $C(0)$ is strongly influenced by the molecular-scale interactions between the material comprising or adhering to the surface of the tube wall and the specific trace gas. A full description of these molecular-scale interactions for any particular atmospheric trace gas likely to be susceptible to surface related sorption and desorption processes (e.g., $\mathrm{H}_{2} \mathrm{O}, \mathrm{NH}_{3}$, $\mathrm{O}_{3}, \mathrm{SO}_{2}$ ) is well beyond the scope of this study. Fortunately though, it is possible to develop a model of the bulk processes sorption/desorption at the tube wall, which can be combined with Eq. (18) to produce a physically realistic, useful, and insightful model of the wall boundary condition.

\subsubsection{Surface sorption/desorption mass flux}

This section develops a model of $C^{\prime}(0)$ that describes the bulk molecular-scale absorption/desorption at the interior surface of a tube wall and derives an empirical, but physically-based, parameterization of the associated (dimensionless) conductance $\left(\kappa_{s}\right)$, which is the sorption/desorption analog of $\kappa_{b}$ above.

We begin with the Hertz-Knudsen-Schrage (HKS) equation, which has its origins in the kinetic theory of gases and describes the net flux of a gas that is simultaneously condensing on and evaporating from a surface. It is used to model water vapor fluxes to and from cloud and ice droplets (e.g., Pruppacher and Klett, 1997; Seinfeld and Pandis, 1998; Marek and Straub, 2001; Li et al., 2001) and the net heat transfer in steam-laden heat pipes and thermosiphons (e.g., Carey, 1992; Fagri, 1995). The HKS equation is

$$
\begin{aligned}
& F_{C, \text { net }}=\frac{2}{2-\mathcal{K}_{c}} \times \\
& {\left[\mathcal{K}_{c} C(0) \sqrt{\frac{R T_{g}}{2 \pi M}}-\mathcal{K}_{e} C_{\mathrm{sat}}\left(T_{l, s}\right) \sqrt{\frac{R T_{l, s}}{2 \pi M}}\right]}
\end{aligned}
$$

where $\mathcal{K}_{c}$ is the condensation coefficient of the gas (sometimes also called the thermal accommodation coefficient), $\mathcal{K}_{e}$ is its evaporation coefficient, $R$ is the universal gas constant, $T_{g}$ is the temperature of the condensing gas, $T_{l, s}$ is the temperature of the evaporating surface-bound molecules (which would be liquid water in the case of water vapor), $C_{\text {sat }}$ is the saturation density of the gas, and $M$ is the molecular mass of the gas. The first term in the brackets on the right hand side of Eq. (19) is condensing mass flux and the second term is the evaporating mass flux. (Note: the HKS equation is usually expressed in terms of the vapor pressure rather than vapor density. Here we have used the ideal gas law to convert the usual HKS equation to the form given above.)

Although Eq. (19) is appropriate for a freely evaporating/condensing gas, to use it to describe surface adsorption/desorption requires introducing the possibility that there are only a finite number of sites available for adsorption (e.g., Silbey et al., 2005). This yields:

$$
\begin{aligned}
& F_{C, \text { net }}=\frac{2}{2-\mathcal{K}_{c}} \times \\
& {\left[\mathcal{K}_{c} C(0) \sqrt{\frac{R T_{g}}{2 \pi M}} \Theta_{c}-\mathcal{K}_{e} C_{\mathrm{sat}}\left(T_{l, s}\right) \sqrt{\frac{R T_{l, s}}{2 \pi M}} \Theta_{e}\right]}
\end{aligned}
$$

where $\Theta_{e}$ is the fraction of the total number of surface absorbing sites covered by adsorbed molecules and $\Theta_{c}$ is the fraction of the total number of sites available for adsorption. $\Theta_{e}$ is usually described by an adsorption isotherm (e.g., Do, 1998). For example, assuming equilibrium conditions (i.e., rate of adsorption $=$ rate of desorption or $F_{C \text {,net }} \equiv 0$ ) and that the absorbate forms a molecular monolayer (i.e., $\Theta_{e}+\Theta_{c}=1$ ), then Eq. (20) yields a variant of the Langmuir adsorption isotherm (e.g., Silbey et al., 2005). For multilayer sorption/desorption there are a number of empirical expressions that are used for the adsorbtion equilibrium isotherm, notable among these is the BET equation, (e.g., Do, 1998). Nevertheless, further discussion of the adsorption isotherm is deferred until after the development of $\kappa_{s}$.

Next are two simplifying assumptions to Eq. (20). The first is to assume that $\mathcal{K}_{c} \ll 1<2$, in accordance with virtually all observational data (Pruppacher and Klett, 1997; Marek and Straub, 2001). Consequently, $2 /\left(2-\mathcal{K}_{c}\right) \approx 1$. The second is to assume that the heat transfer that occurs during surface evaporation and condensation is negligible, which is reasonable for dilute gases and very small sorption and desorption fluxes, such as might be expected for closed-path eddy covariance systems. Consequently, $T_{l, s} \equiv T_{g}$ is assumed. Nevertheless, it is also worth noting that studies of thin film evaporation of water have indicated that the modeled temperature differences between evaporating and condensing molecules can be $30 \mathrm{~K}$ (e.g., Yang and Pan, 2005). As a result this assumption may become suspect at very high humidities and vapor pressures, for which there is the potential to exchange large numbers of water vapor molecules between the tube wall and the free air stream. Again assuming that $F_{C \text {, net }}$ equals the diffusive flux. Equation (20) can now be written in terms of $C^{\prime}(0)$. 


\subsubsection{Synthesizing the wall boundary condition}

$C^{\prime}(0)=\mathcal{K}_{c} S_{c} \frac{a}{v} \sqrt{\frac{R T_{g}}{2 \pi M}} \Theta_{c}\left\{C(0)-\frac{\mathcal{K}_{e} \Theta_{e}}{\mathcal{K}_{c} \Theta_{c}} C_{\mathrm{sat}}\right\}$

The dimensionless wall sorption/desorption conductance is identified from this last relationship as $\kappa_{s}=\mathcal{K}_{c} S_{c}(a / \nu) \sqrt{\left(R T_{g}\right) /(2 \pi M)} \Theta_{c}$. From the kinetic theory of gases the term $\sqrt{\left(R T_{g}\right) /(2 \pi M)}$ is related to the mean (thermal) velocity of a molecule of the gas (which is different from $U(\rho))$, so that $(a / \nu) \sqrt{\left(R T_{g}\right) /(2 \pi M)}$ can be identified as a molecular-scale Reynolds number, $R e_{m}$. Therfore, $\kappa_{s}=\mathcal{K}_{c} R e_{m} S_{c} \Theta_{c}$.

Eliminating $C(0)$ from Eqs. (18) and (21) yields the following expression for $C^{\prime}(0)$ :

$C^{\prime}(0)=\frac{\kappa_{b} \kappa_{s}}{\kappa_{b}+\kappa_{s}}\left\{\frac{C_{\infty}}{C_{\mathrm{sat}}}-\frac{\mathcal{K}_{e} \Theta_{e}}{\mathcal{K}_{c} \Theta_{c}}\right\} C_{\mathrm{sat}}$

But before this equation can be used for the wall boundary condition some adaptation is still necessary.

\subsubsection{Semi-empirical model of the wall boundary condition}

(a) The term $C_{\infty} / C_{\text {sat }}$ can be reasonably approximated by the time-mean relative humidity inside the tube, $\widetilde{h}_{t}$, which can be related to the time-mean ambient atmospheric humidity, $\widetilde{h}$, by accounting for the pressure drop inside the tube. More specifically $C_{\infty} / C_{\mathrm{sat}} \approx \widetilde{h}_{t}=\widetilde{h} \widetilde{p}_{t} / \widetilde{p}_{a}$, where $\widetilde{p}_{t}$ is the time-mean tube pressure or mean internal pressure of the sampler and $\tilde{p}_{a}$ is the time-mean ambient pressure.

(b1) The term $\mathcal{K}_{e} / \mathcal{K}_{c}$ is difficult to know precisely because it depends on the nature of the absorbing surface. For example, for a clean homogenous surface it might be reasonable to assume that $\mathcal{K}_{e} / \mathcal{K}_{c} \approx 0.8$ (Marek and Straub, 2001). But for a tube with an inside surface contaminated with a variety of atmospheric aerosols and therefore condensation nuclei (e.g., Forslund and Leygraf, 1997), as one might expect even with a closed-path eddy covariance tube that includes an inlet filter, then $\mathcal{K}_{e} / \mathcal{K}_{c} \ll 1$ is about all that can be anticipated (Marek and Straub, 2001). Another source of uncertainty in the term $\mathcal{K}_{e} / \mathcal{K}_{c}$ is that it is likely to be a function of temperature because the activation energies for surface adsorbtion and desorption will not necessarily the same (e.g., Silbey et al., 2005; Davidovits et al., 2006).

(b2) The term $\Theta_{e} / \Theta_{c}$ is at least as uncertain as $\mathcal{K}_{e} / \mathcal{K}_{c}$, but for different reasons. Primary among these is the expectation that the number of adsorbing/desorbing sites is likely to be relatively close to steady state for the mean concentration of water vapor, but potentially dynamic in time and location, in regards to water vapor fluctuations inside the tube. To keep the complexity of the model as minimal as possible this term is assumed to be better represented by mean conditions and that the mean sorption/desorption isotherm can be described by the Langmuir isotherm. This means that near equilibrium $\Theta_{e} / \Theta_{c} \gg 1$ (most avaliable surface sorption sites are occupied) and $\Theta_{e} / \Theta_{c} \propto \tilde{h} \tilde{p}_{t} / \tilde{p}_{a}$. Nonetheless, when applying these results to water vapor fluctuations inside the tube, one must allow for the possibility that at any given location there may be a lag time between sorption and desorption and at any given time sorption and desorption may be occuring simultaneously, but at different locations. This suggests that $\Theta_{e} / \Theta_{c}$ should be parameterized to allow for a phase between sorption and desorption. Therefore, it is necessary to allow for the possibility that the model parameter $\Theta_{e} / \Theta_{c}$ may be complex.

(c) Combining (a), (b1), and (b2) suggests the following parameterization:

$\left\{\frac{C_{\infty}}{C_{\mathrm{sat}}}-\frac{\mathcal{K}_{e} \Theta_{e}}{\mathcal{K}_{c} \Theta_{c}}\right\} \approx \widetilde{h} \frac{\widetilde{p}_{t}}{\widetilde{p}_{a}}\left(1+\gamma_{1} e^{i \phi}\right)=\gamma_{*} \tilde{h}$

where $\gamma_{1}$ is a real-valued constant, $\phi$ is the sorption/desorption phase, and $\gamma_{*}$ is a complex-valued parameter defined by the right hand equality of this last expression.

Next $\kappa_{s}$ is parameterized.

(d) $\mathcal{K}_{c}$ depends on the nature of the surface, the sorbent, the nature of the liquid surface or layer formed by the condensed sorbent, and the ambient conditions (e.g., Awakuni and Calderwood, 1972; Andrews and Larson, 1993; Forslund and Leygraf, 1997). For water vapor it is a function both of temperature and pressure and other characteristics of the water surface formed by the adsorbed water vapor (Marek and Straub, 2001; Li et al., 2001). These last two studies suggest that for application to closed path eddy covariance systems it seems reasonable to assume that $10^{-4}<\mathcal{K}_{c}<10^{-1}$.

(e) $R e_{m} \approx(4-6) \times 10^{4}$ for typical ambient temperature and pressures encountered at eddy covariance sites.

(f) Decomposing $\Theta_{c}$ into a mean and fluctuating part yields $\Theta_{c}=\widetilde{\Theta}_{c}+\Delta \Theta_{c} e^{i \omega(t-\lambda x / \bar{U})}$ along with the concomitant assumption that $\Delta \Theta_{c} \ll \widetilde{\Theta}_{c}$. This allows the fluctuating portion of the term $\kappa_{b} \kappa_{s} /\left(\kappa_{b}+\kappa_{s}\right)$ to be linearized such that $\kappa_{b} \kappa_{s} /\left(\kappa_{b}+\kappa_{s}\right) \sim\left[\kappa_{b} \widetilde{\kappa}_{s} /\left(\kappa_{b}+\widetilde{\kappa}_{s}\right)\right]\left[\Delta \Theta_{c} / \widetilde{\Theta}_{c}\right]$ and $\widetilde{\kappa}_{s}=\mathcal{K}_{c} R e_{m} S_{c} \widetilde{\Theta}_{c}$. Although this approach may linearize the dimensionless conductance, in fact there is no other justification for assuming that $\Delta \Theta_{c} \ll \widetilde{\Theta}_{c}$ because the exact relationship between $\Delta \Theta_{c}$ and $\widetilde{\Theta}_{c}$ cannot be known. Other relations may be possible, but we wish to keep the model complexity to a minimum. Next $\Delta \Theta_{c} / \widetilde{\Theta}_{c}$ is assumed to be proportional to $e^{l_{*} \widetilde{h}}$ with $l_{*}>0$. This is purely an empirical parameterization, justified heuristically from observations that the attenuation of water vapor fluctuations increase nonlinearly with increasing humidity (e.g., Peters et al., 2001; Clement, 2004; Ibrom et al., 2007). Note: other mathematical functions, such as those suggested by the BET equation and similar algebraic forms derived to describe multilayer adsorption isotherms (e.g., Do, 1998), could have been used instead of the exponential form $e^{l_{*} \widetilde{h}}$. But, such algebraic forms may not be very useful or even physically realistic at high humidities because they become mathematically undefined at $\widetilde{h}=1$. 


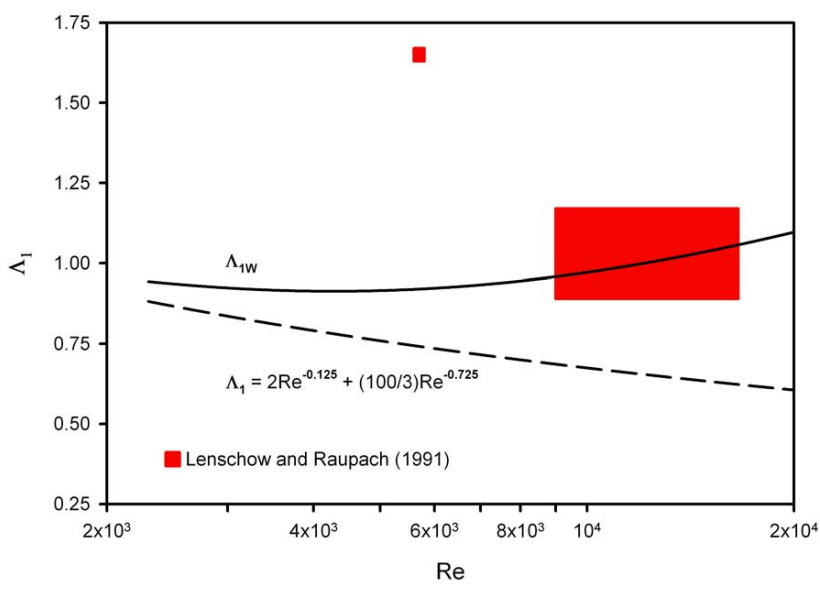

Fig. 3. Transfer function attenuation coefficients: $\Lambda_{1 W}$ for water vapor modeled with the wall boundary condition (Eq. 24), the analytical approximation for a passive tracer (shown in Fig. 2), and a summary of some experimental observations of the attenuation coefficient for water vapor fluctuations from Lenschow and Raupach (1991) as determined by Massman (1991).

(g) The saturation density, $C_{\text {sat }}$, is a function of the gas temperature, $T_{g}$, and can be expressed as $C_{\text {sat }}\left(T_{0}\right) f\left(T_{g}\right)$, where $T_{0}$ is $0 \mathrm{C}$ (or $273.15 \mathrm{~K}$ ) and $f\left(T_{g}\right.$ ) is the function that describes the behavior of $C_{\text {sat }}$ when $T_{g}>0 \mathrm{C}$.

Combining (d) through (g) with (c), Eq. (22) suggests the following empirical models of the wall boundary condition for the eigenfunctions $\widehat{C}_{\lambda}(\rho)$ and $\widehat{C}_{n}(\rho)$ :

$$
\begin{aligned}
& \widehat{C}_{\lambda}^{\prime}(0)=\left[\frac{\kappa_{b} \widetilde{\kappa}_{s}}{\kappa_{b}+\widetilde{\kappa}_{s}}\right] \gamma_{*} \tilde{h} e^{l_{*} \tilde{h}} \\
& \widehat{C}_{n}^{\prime}(0)=(i)^{n}\left[\frac{\kappa_{b} \widetilde{\kappa}_{s}}{\kappa_{b}+\widetilde{\kappa}_{s}}\right] \gamma_{*} \tilde{h} e^{l_{*} \tilde{h}}
\end{aligned}
$$

where $\gamma_{*}$ is now an adjustable empirical parameter that subsumes all the many uncertain and unspecified (and possibly un-specifiable) physical processes, relationships, and dependencies discussed above. Note there is no loss of generality by neglecting $C_{\text {sat }}\left(T_{0}\right)$ in Eqs. (23) and (24). This is equivalent (mathematically) to defining the eigenfunction (which is dimensionless) in terms of $C(\rho) / C_{\text {sat }}\left(T_{0}\right)$. In this way $C_{\text {sat }}\left(T_{0}\right)$ is simply a scaling factor that has no impact on the eigenvectors, the eigenvalues, or the transfer function. Also note that Eqs. (23) and (24) are related, but they are not mathematically equivalent to one another.

This section closes with a numerical estimation of $\widetilde{\kappa}_{s}$. Combining (d), (e), and the expectation that $\widetilde{\Theta}_{c} \ll 1$ suggests that it is reasonable to assume $\widetilde{\kappa}_{s} \sim \mathcal{O}(1)$. But it is possible to improve on this estimate of $\widetilde{\kappa}_{s}$ by determining the expected range of values for $\kappa_{b}$, because it is unlikely that $\widetilde{\kappa}_{s}$ is small compared to $\kappa_{b}$, otherwise the wall flux will begin to become negligibly small (which is equivalent to assuming a passive tracer). Therefore, it is reasonable to expect that $\widetilde{\kappa}_{s} \sim \kappa_{b}$ or $\widetilde{\kappa}_{s}>\kappa_{b}$. The definition for $\kappa_{b}$ (above) yields $0.25<\kappa_{b}<4.75$. With these results as guidance, and largely for computational purposes, $\widetilde{\kappa}_{s}=1$ is assumed.

\subsection{Modeling results}

Figure 3 compares the attenuation coefficient for the turbulent tube flow model with a sorbing/desorbing wall boundary condition, $\Lambda_{1 W}$, with the observations of Lenschow and Raupach (1991) and with $2 R e^{-1 / 8}+(100 / 3) R e^{-0.725}$, the approximation to $\Lambda_{1}$ for the case that water vapor is considered a passive tracer (Fig. 2). These calculations for $\Lambda_{1 W}$ assume that $\widetilde{\kappa}_{s}=1$ (see preceeding discussion), $l_{*}=8.26$ (obtained by fitting the humidity/cutoff-frequency data of Ibrom et al. (2007)), $\widetilde{h}=0.2$ (Lenschow, personal communication, 2007), and $\gamma_{*}=0.4$ (chosen to approximate the data of Lenschow and Raupach, 1991, within the flow region $9000 \leq R e \leq 16600$ ). Note choosing $\gamma_{*}$ as a real-valued parameter, rather than a complex-valued one, does not imply any obscure assumptions about the possible phase, $e^{i \phi}$, or value of the parameter $\gamma$, both of which are discussed above in regards to $\Theta_{e} / \Theta_{c}$. In theory the complex part of $\gamma_{*}$ can be estimated from observing how the nominal tube lag time, $L / \bar{U}$, might vary with frequency (the $\Lambda_{2}$ term of the transfer function), but there is not enough observational evidence to support exploring this possibility.

The main conclusion to be drawn from Fig. 3 is that the wall boundary condition, as formulated by Eq. (24), predicts that the attenuation of water vapor fluctuations should increase with increasing $R e$, which according to Lenschow and Raupach (1991) and Massman (1991) they do not. Thus we must conclude that either (1) variations in ambient humidity during the experiments of Lenschow and Raupach (1991) are causing the apparent $R e$ dependency or (2) something is missing from the model wall boundary condition. One possibility for the latter is that the probability of a molecule being captured by (or condensing on) the interior tube surface is dependent upon the tube flow velocity, such that a molecule is more likely to be captured when the tube flow rate is slower than when it is faster. This would imply that $\widetilde{\kappa}_{s} \propto R e^{-n}$ or more likely that $\widetilde{\kappa}_{s} \propto R e^{-n} \mathrm{Sc}^{-m}$, where $n, m>0$. But this is speculation only and further speculations on this point are beyond the scope and intent of the present study and so will not be pursued here. Nonetheless, we assume that the present results clearly indicate a need to formulate the boundary condition to include some, otherwise heuristic, dependency on $R e^{-n}$.

\subsection{Empirical adjustments and analytical simplifications}

We did explore the possibility of parameterizing $\widetilde{\kappa}_{s} \propto R e^{-n}$, but found that although such a formulation for the boundary condition did capture some of the observed $\Lambda_{1} \propto R e^{-n}$ dependency, it was not fully satisfactory. On the other hand, 
further trial and error did yield the following (quite satisfactory) formulation for the wall boundary condition:

$\widehat{C}_{n}^{\prime}(0)=(i)^{n}\left[\frac{\kappa_{b} \widetilde{\kappa}_{s}}{\kappa_{b}+\widetilde{\kappa}_{s}}\right]\left[(\operatorname{Re} / 1000)^{-4} \gamma_{*}\right] \tilde{h} e^{l_{*} \tilde{h}}$

with $\gamma_{*}=1760$ and all other parameters are as before. The resulting $\Lambda_{1 W}$ is shown as a function of $R e$ in Fig. 4 and it clearly compares very well with the data of Lenschow and Raupach (1991).

Also shown in Fig. 4 is the following analytical expression for $\Lambda_{1 W}$, which captures most of the numerical results derived with the tube flow model and boundary condition provided by Eq. (25).

$$
\begin{gathered}
\Lambda_{1 W}=2 R e^{-1 / 8}+G_{0} R e^{-0.725}+ \\
G_{1}\left[(\operatorname{Re} / 1000)^{-3}\right] \tilde{h} e^{l_{*} \tilde{h}}
\end{gathered}
$$

where in general $G_{0}, G_{1}$, and $l_{*}$ are empirically-determined coefficients. For this study $G_{0}=100 / 3$ and $G_{1}=100 S c^{-1 / 2}$, which were determined by (visually) fitting the Lenschow and Raupach data shown in Fig. 4.

Equation (26) summarizes the results of the eigenvalue model with a simpler and more concise formulation for $\Lambda_{1 W}$. Although it can be used in conjuction with the transfer function, Eq. (9), to provide initial estimates of (and spectral corrections for) the attenuation of water vapor fluctuations by closed-path eddy covariance systems, it can be further simplified, which should make it more useful for any given site.

\subsection{Some practical matters}

Equation (26) is most applicable to a straight horizontal tube, but closed-path flux systems are rarely implemented as such. In general, departures from the ideal tube will tend to increase the attenuation (e.g., Lenschow and Raupach, 1991), which suggests that the present attenuation model for sorption/desorption at the tube wall (Eqs. 9 and 26) may need to be calibrated against spectra of trace gases on a site specific basis. (Note this is basically how Massman (1991)'s original model is often used for passive tracers, except, of course, that for his model $G_{1} \equiv 0$.)

This suggests replacing $\Lambda_{1 W}$ above with a more convenient expression, such as,

$\Lambda_{1 W}=G_{0}+G_{1} \tilde{h} e^{l_{*} \tilde{h}}$

which follows from Equation (26) by assuming that the tube Reynolds number, $R e$, is a fixed parameter or characteristic of any given installation. Unfortunately there remains the (previously mentioned) possibility that temperature and other effects may cause temporal variations in $R e$ and, therefore, in $G_{0}$ and $G_{1}$ (Eq. 27). Such variation in the parameters can be observed or deduced from variations in the attenuation of spectra of trace gases obtained with a closed-path system.

At the beginning of this paper we suggested that our modeling efforts might prove insightful for measuring isotope

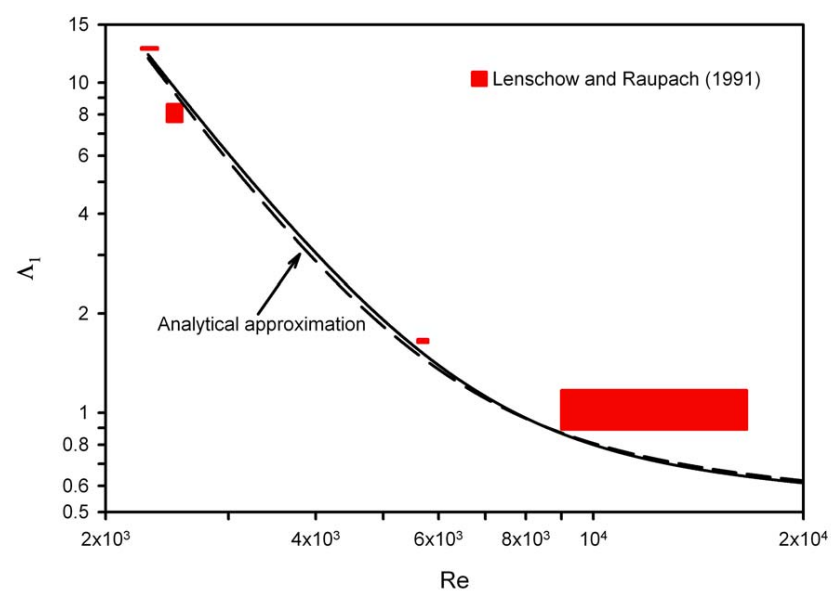

Fig. 4. Transfer function attenuation coefficients $\Lambda_{1 W}$ for water vapor modeled with the wall boundary condition (Eq. 25), modeled by its analytical approximation (Eq. 26), and a summary of some experimental observations of the attenuation coefficient for water vapor fluctuations from Lenschow and Raupach (1991) as determined by Massman (1991).

fluxes with closed-path systems. Although it is possible to simply employ Eqs. (9) and (26) or (27) to describe tube flow attenuation of isotopes, this may not be the best way to model the isotopic fractionation effects of wall sorption for (at least) three reasons. First, a much better approach would employ laminar tube flow ( $R e \leq 2000$, approximately), which maximizes the fractionation effects (as opposed to turbulent flow, which will minimize these effects) because transport by molecular diffusivity is much more discriminating than turbulent diffusion. But in the case of laminar tube flow the transfer function for describing high frequency attenuation is not same as that suggested by Eq. (9) (e.g., see the case $\Omega \gg 1$ discussed by Barton, 1983; Stokes and Barton, 1990). Second, the present model does not necessarily capture the Schmidt number dependency very well. In the preceeding section we proposed that $G_{1} \sim S c^{-1 / 2}$ primarily for convenience, whereas there is no a priori justification for assuming that the exponent is $-1 / 2$. Finally, the wall boundary condition should not be taken as fully satisfactory or final, so other formulations and models need to be explored and developed. Given these (three) concerns, we suggest that the insights gained from the present study be used in a separate study of laminar tube flow and boundary conditions to address the issue of fractionation of isotopes of water vapor by wall sorption. Furthermore, any observational study of isotopic fractionation would likely result in some significant insights into the physics of wall sorption and the wall boundary condition. 


\section{Conclusions}

This study takes a fresh look at the attenuation of fluctuations of scalars in turbulent flow inside sampling tubes. We re-examine some old results for passive scalars and propose a new physically-based formulation to describe the attenuation of water vapor fluctuations for use with closed-path eddy covariance systems. The fact that both Taylor (1954)'s model and the present eigenvalue model, (Eq. 3 plus the new formulations for the turbulent diffusivity $D(\rho)$ ) yield very similar results for the first order attenuation coefficient $\Lambda_{1}$ tends to support the notion that the physical basis of each model is reasonably correct. The benefit of the eigenvalue model is that it provides for more detail about radial diffusion and the effects of the momentum boundary layer on the both $\Lambda_{1}$ and the second order attenuation coefficient $\Lambda_{2}$. Nevertheless, neither of these two models, nor the separation-of-variables model (which predicted much less attenuation than the other two models), was successful at explaining the attenuation of water vapor fluctuations observed by Lenschow and Raupach (1991). This leads to the conclusion that water vapor is not a passive scalar and that sorption/desorption of water vapor must have been occuring at the tube walls during the experiments of Lenschow and Raupach (1991). On the other hand, perhaps this conclusion should not be surprising given recent observations that the attenuation of water vapor fluctuations in sampling tubes are strongly and nonlinearly dependent upon humidity (e.g., Clement, 2004; Amman et al., 2006; Ibrom et al., 2007). Beginning with the
Hertz-Knudsen-Schrage equation (e.g., Marek and Straub, 2001), this study takes a step-by-step approach to formulating a flux boundary condition at the tube wall that describes the sorption/desorption of molecules at the wall surface in turbulent tube flow. The boundary condition is formulated in accordance with the observed non-linear dependency on humidity. Although the sorption/desorption model did capture the humidity effects and did predict greater attenuation than the passive scalar model, it still did not fully describe the results of Lenschow and Raupach (1991)! The most immediate cause of failure of this model (or of the boundary condition) is that the (often-studied, well-known) turbulent boundary layer resistance to mass transfer suggests that the attenuation should increase with Reynolds number, which is contrary to Lenschow and Raupach (1991). Empirical adjustments to the boundary condition did improve the model and did capture the data of Lenschow and Raupach (1991). In turn this allowed the derivation of a single analytical expression of the attenuation of coefficient $\Lambda_{1}$ that also captured the Reynolds number dependency of Lenschow and Raupach (1991), as well as the humidity dependency of Ibrom et al. (2007). We hypothesized that the sorption/desorption fluxes, or more specifically the number of sorption/desorption sites at the tube wall, could be dependent upon the Reynolds number in a way that can account for the data of Lenschow and Raupach (1991). But, the physical basis for such a phenomenon is unknown (at least to the authors). 


\section{List of symbols}

\begin{tabular}{|c|c|c|}
\hline$a$ & $=$ & tube radius \\
\hline$h_{C}$ & $=$ & dimensionless wall turbulent transfer coefficient \\
\hline$h(\omega)$ & $=$ & complex-valued tube transfer function \\
\hline$\underset{\sim}{h_{\text {phase }}}(\omega)$ & $=$ & phase shift transfer function \\
\hline$\widetilde{h}$ & $=$ & mean atmospheric relative humidity \\
\hline$l_{*}$ & $=$ & adjustable empirical coefficient for wall sorption \\
\hline$r$ & $=$ & tube radial coordinate \\
\hline$t$ & $=$ & time \\
\hline$u_{*}$ & $=$ & wall friction velocity \\
\hline$x$ & $=$ & tube longitudinal coordinate \\
\hline$A$ & $=$ & parameter for van Driest form of turbulent diffusivity \\
\hline$B$ & $=$ & parameter for Reichardt form of turbulent diffusivity \\
\hline$C=C(r, x, t)$ & $=$ & solute mass concentration \\
\hline $\bar{C}=C(x, t)$ & $=$ & cross-sectionally averaged concentration \\
\hline$\widehat{C}_{\lambda}(\rho)$ & $=$ & eigenfunction form of solute mass concentration \\
\hline$\widehat{C}_{n}(\rho)$ & $=$ & harmonic decomposition of $\widehat{C}_{\lambda}(\rho)$ \\
\hline$C_{\text {sat }}$ & $=$ & saturation value of $C$, a function of temperature \\
\hline$C^{\prime}(0)=\{d C / d \rho\}_{\rho=0}$ & $=$ & derivative of $C(\rho)$ at the tube wall \\
\hline$D(r)$ and $D(\rho)$ & $=$ & radial profile of turbulent diffusivity \\
\hline$D(1)$ & $=$ & turbulent diffusivity at the tube centerline \\
\hline $\bar{D}$ & $=$ & cross-sectionally averaged turbulent diffusivity \\
\hline$E(\rho)$ & $=$ & dimensionless Reichardt's turbulent diffusivity \\
\hline$F(\rho)$ & $=$ & dimensionless auxiliary variable, related to $D(\rho)$ \\
\hline$F_{C}$ & $=$ & adsorption/desorption flux of solute at the tube wall \\
\hline$G(\rho)$ & $=$ & dimensionless velocity profile for turbulent tube flow \\
\hline$G_{D}(\rho)$ & $=$ & normalized turbulent diffusivity profile \\
\hline$G_{U}(\rho)$ & $=$ & normalized velocity profile \\
\hline$G_{0} ; G_{1}$ & $=$ & adjustable empirical coefficients \\
\hline$H(\omega)$ & $=$ & transfer function for attenuation of fluctuations \\
\hline$K=a u_{*} / v$ & $=$ & Karman number \\
\hline $\mathcal{K}_{c}$ & $=$ & wall condensation (adsorption) coefficient \\
\hline $\mathcal{K}_{e}$ & $=$ & wall evaporation (desorption) coefficient \\
\hline$L$ & $=$ & tube length \\
\hline$M$ & $=$ & molecular mass \\
\hline$R$ & $=$ & universal gas constant \\
\hline $\operatorname{Re}$ & $=$ & $2 a \bar{U} / \nu=$ tube flow Reynolds number \\
\hline$S c$ & $=$ & Schmidt number \\
\hline$T$ & $=$ & temperature (degrees Kelvin) \\
\hline$U(r)$ and $U(\rho)$ & $=$ & radial profile of flow velocity \\
\hline $\bar{U}$ & $=$ & cross-sectionally averaged turbulent tube flow velocity \\
\hline$V(\rho)$ & $=$ & van Driest function: Reynolds stress near tube wall \\
\hline$\gamma$ & $=$ & flow related parameter $=4 D^{2}(1) v^{-2} R e^{-2}$ \\
\hline$\gamma_{1}$ & $=$ & adjustable empirical coefficient \\
\hline$\gamma_{*}$ & $=$ & adjustable empirical coefficient, related to $\gamma_{1}$ \\
\hline$\kappa_{b}$ & $=$ & dimensionless wall boundary layer conductance \\
\hline$\kappa_{S}$ & $=$ & dimensionless wall adsorption/desorption conductance \\
\hline$\lambda$ & $=$ & eigenvalue; see Eq. (2) \\
\hline$v$ & $=$ & molecular viscosity of air \\
\hline$v_{T}(\rho)$ & $=$ & turbulent viscosity for tube flow \\
\hline$\rho=(1-r / a)$ & $=$ & dimensionless radial (or wall) coordinate \\
\hline$\omega$ & $=$ & frequency $\left[\right.$ radians s $\left.{ }^{-1}\right]$ \\
\hline$\Delta_{D}$ & $=$ & model parameter defined by $D(1) / \nu=R e^{7 / 8} \Delta_{D}$ \\
\hline$\Theta_{c}$ & $=$ & relative measure of tube wall condensation sites \\
\hline$\Theta_{e}$ & $=$ & relative measure of evaporation sites on tube wall \\
\hline$\Lambda_{1} ; \Lambda_{2}$ & $=$ & attenuation coefficients, related to $\lambda$ \\
\hline$\Lambda_{1 T} ; \Lambda_{2 T}$ & $=$ & attenuation coefficients for Taylor's model \\
\hline$\Omega$ & $=$ & dimensionless frequency $=a^{2} \omega / D(1)$ \\
\hline$\Omega_{T}$ & $=$ & dimensionless frequency for Taylor's model \\
\hline
\end{tabular}


Acknowledgements. Partial support for this study was provided to the first author by the EU-project ACCENT and Ris $\emptyset$ National Laboratory (DTU). I (wjm) would also like to thank the ACCENT and Ris $\varnothing$ staff members for their assistance and generosity and especially L. Kristensen for his encouragement and helpful discussions.

Edited by: V. F. McNeill

\section{References}

Ammann, C., Brunner, A., Spirig, C., and Neftel, A.: Technical Note: Water vapour concentration and flux measurements with PTR-MS, Atmos. Chem. Phys., 6, 4643-4651, 2006, http://www.atmos-chem-phys.net/6/4643/2006/.

Andrews, E. and Larson, S. M.: Effect of surfactant layers on the size changes of aerosol particles as a function of relative humidity, Environ. Sci. Technol., 27, 857-865, 1993.

Aravinth, S.: Prediction of heat and mass transfer for fully developed turbulent fluid flow in tube, Int. J. Heat Mass Tran., 43, 1399-1408, 2000.

Awakuni, Y. and Calderwood, J. H.: Water vapour adsorption and surface conductivity in solids, J. Phys. D Appl. Phys., 5, 10381045, 1972.

Barton, N. G.: The dispersion of solute from time-dependent releases in parallel flow, J. Fluid Mech., 136, 243-267, 1983.

Brutsaert, W.: Evaporation into the Atmosphere, D Reidel Publishing Co., Dordrecht, 299 pp., 1982.

Carey, V. P.: Liquid-Vapor Phase-Change Phenomena: An Introduction to the Thermophysics of Vaporization and Condensation Processes in Heat Transfer Equipment, Hemisphere Publishing Corp., Washington, D.C., 645 pp., 1992.

Clement, R.: Mass and energy exchange of a plantation forest in Scotland using micrometeorological methods, Chapter 5 of $\mathrm{PhD}$ thesis, University of Edinburgh, Scotland, UK, 2004.

Davidovits, P., Kolb, C. E., Williams, L. R., Jayne, J. T., and Worsnop, D. R.: Mass accommodation and chemical reactions at gas-liquid interfaces, Chem. Rev., 106, 1323-1354, 2006.

Do, D. D.: Adsorption Analysis: Equilibria and Kinetics, Imperial College Press, London, 892 pp., 1998.

Fagri, A.: Heat Pipe Science and Technology, Taylor \& Francis, Washington, D.C., 874 pp., 1995.

Forslund, M. and Leygraf, C.: Humidity sorption due to deposited aerosol particles studied in situ outdoors on gold surfaces, J. Electrochem. Soc., 144, 105-113, 1997.

Hussein, H. J., Capp, S., and George, W. K.: Velocity measurements in a high-Reynolds-number, momentum-conserving, axisymmetric, turbulent jet, J. Fluid Mech., 258, 31-75, 1994.

Ibrom, A., Dellwik, E., Flyvbjerg, H., Jensen, N. O., and Pilegaard, K.: Strong low-pass filtering effects on water vapour flux measurements with closed-path eddy covariance systems, Agr. Forest Meteorol., 147, 140-156, 2007.

Kays, W. M. and Crawford, M. E.: Convective Heat and Mass Transfer, McGraw-Hill Inc., New York, 601 pp., 1993.

Kim, J., Moin, P., and Moser, R.: Turbulence statistics in fully developed channel flow at low Reynolds number, J. Fluid Mech., 177, 133-166, 1987.

Kirkegaard, P. and Kristensen, L.: Turbulent flow in tubes: Semianalytic solutions, Z. Angew. Math. Mech., 76, 251-252, 1996.
Lenschow, D. H. and Raupach, M. R.: The attenuation of fluctuations in scalar concentrations through sampling tubes, J. Geophys. Res., 96, 15 259-15 268, 1991.

Li, Y. Q., Davidovits, P., Shi, Q., Jayne, J. T., Kolb, C. E., and Worsnop, D. R.: Mass and thermal accommodation coefficients for $\mathrm{H}_{2} \mathrm{O}(\mathrm{g})$ on liquid water as a function of temperature, J. Phys. Chem. A, 105, 10 627-10 634, 2001.

Marek, R. and Straub, J.: Analysis of the evaporation coefficient and condensation coefficient of water, Int. J. Heat Mass Tran., 44, 39-53, 2001.

Massman, W. J.: The attenuation of concentration fluctuations in turbulent flow through a tube, J. Geophys. Res., 96, $15269-$ $15273,1991$.

Massman, W. J.: A simple method for estimating frequency response corrections for eddy covariance systems, Agr. Forest Meteorol., 104, 185-198, 2002.

Massman, W. J.: Concerning the measurement of atmospheric trace gas fluxes with open- and closed-path eddy covariance systems: The WPL terms and spectral attenuation, in: Handbook of Micrometeorology: A Guide to Surface Flux Measurements, edited by: Lee, X., Massman, W. J., and Law, B. E., Springer, Dordrecht, The Netherlands, 133-160, 2004.

McKeon, B. J., Zararola, M. V., and Smits, A. J.: A new friction factor relationship for fully developed pipe flow, J. Fluid Mech., 538, 429-443, 2005.

Peters, G., Fischer, B., and Münster, H.: Eddy covariance measurements with closed-path optical humidity sensors: A feasible concept?, J. Atmos. Ocean. Tech., 18, 503-514, 2001.

Philip, J. R.: The theory of dispersal during laminar flow in tubes, I, Aust. J. Phys., 16, 287-299, 1963a.

Philip, J. R.: The damping of a fluctuation concentration by continuous sampling through a tube, Aust. J. Phys., 16, 454-463, 1963b.

Pinczewski, W. V. and Sideman, S.: A model for mass (heat) transfer in turbulent tube flow. Moderate and high Schmidt (Prandtl) numbers, Chem. Eng. Sci., 29, 1969-1976, 1974.

Polyanin, A. D., Kutepov, A. M., Vyazmin, A. V., and Kazenin, D. A.: Hydrodynamics, Mass and Heat Transfer in Chemical Engineering, Taylor \& Francis, New York, 386 pp., 2002.

Pope, S. B.: Turbulent Flows, Cambridge University Press, Cambridge, 771 pp., 2000.

Press, W. H., Teukolsky, S. A., Williams, W. T., and Flannery, B. P.: Numerical Recipes, 2nd Edition, Cambridge University Press, Cambridge, 963 pp., 1992.

Pruppacher, H. R. and Klett, J. D.: Microphysics of clouds and Precipitation, Kluwer Academic Publishers, Dordrecht, 954 pp., 1997.

Reichardt, H.: Die grundlagen des turbulenten wärmeüberganges, Archiv gesamte Wärmetechnik, 6/7, 129-143, 1951.

Rusak, Z. and Meyerholz, J.: Mean velocity of fully developed turbulent pipe flows, AIAA Journal, 44, 2793-2797, 2006.

Seinfeld, J. H. and Pandis, S. N.: Atmospheric Chemistry and Physics, John Wiley \& Sons, Inc., New York, 1326 pp., 1998.

Sherwood, T. K., Pigford, R. L., and Wilke, C. R.: Mass Transfer, 3rd Edition, McGraw-Hill, New York, 677 pp., 1975.

Silbey, R. J., Alberty, R. A., and Bawendi, M G.: Physical Chemistry, 4th Edition, John Wiley \& Sons, Inc., New York, 944 pp., 2005.

Stokes, A. N. and Barton, N. G.: The concentration distribution pro- 
duced by shear dispersion of solute in Poiseuille flow, J. Fluid Mech., 210, 201-221, 1990.

Studnikov, E. L.: The viscosity of moist air, J. Engr. Phys. Thermophys., 19, 1036-1037, 1970.

Taylor, G. I.: The dispersion of matter in turbulent flow through a pipe, Proc. R. Soc. Lon. Ser.-A, 223, 446-468, 1954.

Tsilingiris, P. T.: Thermophysical and transport properties of humid air at temperature range between 0 and $100{ }^{\circ} \mathrm{C}$, Energy Conversion and Management, 49, 1098-1110, 2008.

Yang, T. H. and Pan, C.: Molecular dynamics simulations of a thin water layer evaporation and evaporation coefficient, Int. J. Heat Mass Tran., 48, 3516-3526, 2005.

van Driest, E. R.: On turbulent flow near a wall, J. Aeronaut. Sci., 23, 1007-1036, 1956. 